\title{
Abundancia estival de aves costeras y mamíferos marinos en islas Desertores, comuna de Chaitén (Patagonia chilena)
}

\author{
Summer diversity of coastal birds and marine mammals in \\ Desertores Islands, Chaitén commune (Chilean Patagonia)
}

Claudio Delgado ${ }^{1}$, Jaime A. Cursach ${ }^{1} \&$ José Cárdenas-Véjar ${ }^{1}$

\section{Resumen}

Entre los días 24 al 27 de enero de 2019, se navegó el mar de islas Desertores, comuna de Chaitén, en la región de Los Lagos, sur de Chile. Esto con el objetivo de i) estimar la riqueza y abundancia de aves y mamíferos marinos del área de influencia de estas islas, junto con ii) identificar sitios de reproducción. Así, se realizaron registros sobre riqueza, abundancia y distribución espacial de las aves y mamíferos marinos observados durante la navegación, como así también mediante recorridos a pie en islote Nihuel $\left(42,626^{\circ} \mathrm{S} ; 72,933^{\circ} \mathrm{O}\right)$ e islote Barraco $\left(42,737^{\circ} \mathrm{S} ; 73,065^{\circ} \mathrm{O}\right)$. Se determinó que las especies gaviota cahuil (Chroicocephalus maculipennis), cormorán imperial (Leucocarbo atriceps), gaviota dominicana (Larus dominicanus) y blanquillo (Podiceps occipitalis), fueron las aves costeras más abundantes. Mientras que el lobo marino común (Otaria flavescens) y delfín austral (Lagenorhynchus australis), fueron los mamíferos marinos más abundantes. A nivel espacial, las mayores concentraciones de aves y mamíferos marinos se encontraron en los islotes Nihuel y Barraco, como así también en el litoral de isla Talcán. En el islote Nihuel nidifican al menos 2.100 parejas de cormorán imperial, lo que convierte a este lugar en uno de los sitios reproductivos más importantes para dicha especie en Chile. Así también, existe una colonia reproductiva de lobo fino austral (Arctocephalus australis), compuesta por al menos 890 individuos. Por otra parte, el islote Barraco es utilizado por gaviotines sudamericanos (Sterna hirundinacea) como sitio de nidificación, junto a un interesante grupo de aves playeras migratorias.

\section{Palabras clave:}

conservación, ecorregión Chiloense, nidificación, sitios reproductivos.

\section{Abstract}

Between 24th and 27th January of 2019, was sailed the sea of Desertores Islands, commune of Chaitén, region of Los Lagos, southern Chile. This with the objective of i) estimating the richness and abundance of birds and marine mammals in the area of influence of these islands, together with ii) identifying breeding sites. So, records were made on the richness, abundance and spatial distribution of birds and marine mammals observed during navigation, and also walking on Nihuel islet $\left(42.626^{\circ} \mathrm{S} ; 72.933^{\circ} \mathrm{W}\right)$ and Barraco islet $\left(42.737^{\circ} \mathrm{S} ; 73.065^{\circ} \mathrm{W}\right)$. It was determined that the species Brown-hooded gull (Chroicocephalus maculipennis), Imperial cormorant (Leucocarbo atriceps), kelp gull (Larus dominicanus) and Silvery grebe (Podiceps occipitalis), were the most abundant coastal birds. While the South American sea lion (Otaria flavescens) and Peale's dolphin (Lagenorhynchus australis), were the most abundant marine mammals. At the spatial level, the highest concentrations of birds and marine mammals were found in the islets Nihuel and Barraco, as well as on the coast of Talcán Island. On the islet Nihuel nest at least 2100 pairs

Fundación Conservación Marina.

Pérez Rosales 640, oficina 21, Valdivia, Chile.

$\triangle$ jcurval@gmail.com 
of Imperial cormorant, which makes this place one of the most important reproductive sites for this species in Chile. Also, there is a reproductive colony of South American fur seal (Arctocephalus australis), composed of at least 890 individuals. On the other hand, the Barraco islet is used by South American tern (Sterna hirundinacea) as a nesting site, together with an interesting group of migratory shorebirds.

\section{Key words:}

conservation, Chiloense Ecoregion, nesting, reproductive sites.

\section{INTRODUCCIÓN}

La ecorregión Chiloense es un área extensa de mares interiores, fiordos y canales que se caracterizan por ser particularmente productivos y habitados por una importante biodiversidad, ubicándose geográficamente $\left(41^{-} 47^{\circ} \mathrm{S}\right)$ en las regiones de Los Lagos y Aysén, sur de Chile (Sullivan \& Bustamante, 1999; Hucke-Gaete et al. 2010). La significativa productividad primaria (fitoplancton) que se desarrolla de forma estacional en las aguas de la ecorregión sustenta la formación de grandes concentraciones de eufáusidos (comúnmente denominado krill) y langostino de los canales (Munida sp.), los cuales a su vez son el alimento base de muchas especies consumidoras de mayor nivel trófico como aves y mamíferos marinos (Hucke-Gaete et al. 2010).

Dentro de la ecorregión Chiloense, Corcovado $\left(43^{\circ} \mathrm{S}\right)$ destaca por ser un sitio de relevancia para la biodiversidad de aves y mamíferos marinos, residentes y migratorios (Imberti, 2005; Aguayo et al. 2006; Hucke-Gaete et al. 2010; Häussermann et al. 2012). Al norte del golfo de Corcovado se ubica el mar de Chaitén e islas Desertores, que pese a ser contiguo a dicha zona de interés, no existen registros publicados sobre la riqueza y abundancia de aves y mamíferos marinos. Las islas Desertores se ubican en el territorio de la comuna de Chaitén, en la región de Los Lagos, sur de Chile, dentro de un amplio sistema geográfico de bosques milenarios, fiordos y ríos. El borde costero de esta comuna presenta un bajo grado de ocupación humana, razón por la cual predominan paisajes silvestres asociados a zonas frías y a suelos de ñadis (Luebert
\& Pliscoff, 2006; De la Barrera et al. 2011). En su litoral también existen playas de arena y piedras, bordes rocosos, estuarios y humedales costeros, destacando la presencia del grupo islas Desertores.

Islas Desertores constituyen un hito geográfico para la oceanografía local, aflorando desde un mar interior profundo y generando una barrera física que da origen a dos microcuencas submarinas al norte y sur de éstas (Palma et al. 2011). La columna de agua de ambas microcuencas es diferente, con una mayor estabilidad $y$ estratificación en la zona norte, siendo además menos salina en su superficie $(<30 \mathrm{~m}$ ) (Palma \& Silva, 2004; Iriarte et al. 2007). Todo este mar interior presenta una importante producción de clorofila (a) y zooplancton, que permiten sostener redes ecológicas conformadas por una importante y amenazada biodiversidad marina (Palma \& Silva, 2004; Ramírez \& Pizarro, 2005; Iriarte et al. 2007; Hucke-Gaete et al. 2010).

Con la intención de aportar al conocimiento del patrimonio natural que habita en islas Desertores, los objetivos del presente trabajo son a) estimar la riqueza y abundancia de aves y mamíferos marinos del área de influencia de estas islas, junto con b) identificar sitios de reproducción.

\section{MATERIALES Y MÉTODOS}

Entre los días 24 al 27 de enero de 2019, se navegó el mar de islas Desertores, comuna de Chaitén, mediante una lancha a motor con una eslora de $12 \mathrm{~m}$. Se realizaron registros sobre riqueza, abundancia y distribución espacial de las aves y mamíferos marinos observados durante la navegación, como así también mediante recorridos a pie en islote Nihuel $\left(42,626^{\circ} \mathrm{S} ; 72,933^{\circ} \mathrm{O}\right) \mathrm{e}$ islote Barraco $\left(42,737^{\circ} \mathrm{S} ; 73,065^{\circ} \mathrm{O}\right)$ (Fig. 1).

La riqueza y abundancia de aves y mamíferos marinos se determinó mediante transectos de navegación (Tabla 1), adaptando la metodología propuesta por Tasker et al. (1984), en donde tres observadores entrenados realizaron registros en períodos de seis minutos, cada 10 minutos, dentro de una distancia de $300 \mathrm{~m}$ a ambos lados de la embarcación, que se desplazaba a una velocidad promedio de 10 nudos.

Mediante caminata silenciosa se recorrió $(0,5 \mathrm{~km})$ la totalidad del islote Barraco, realizando 


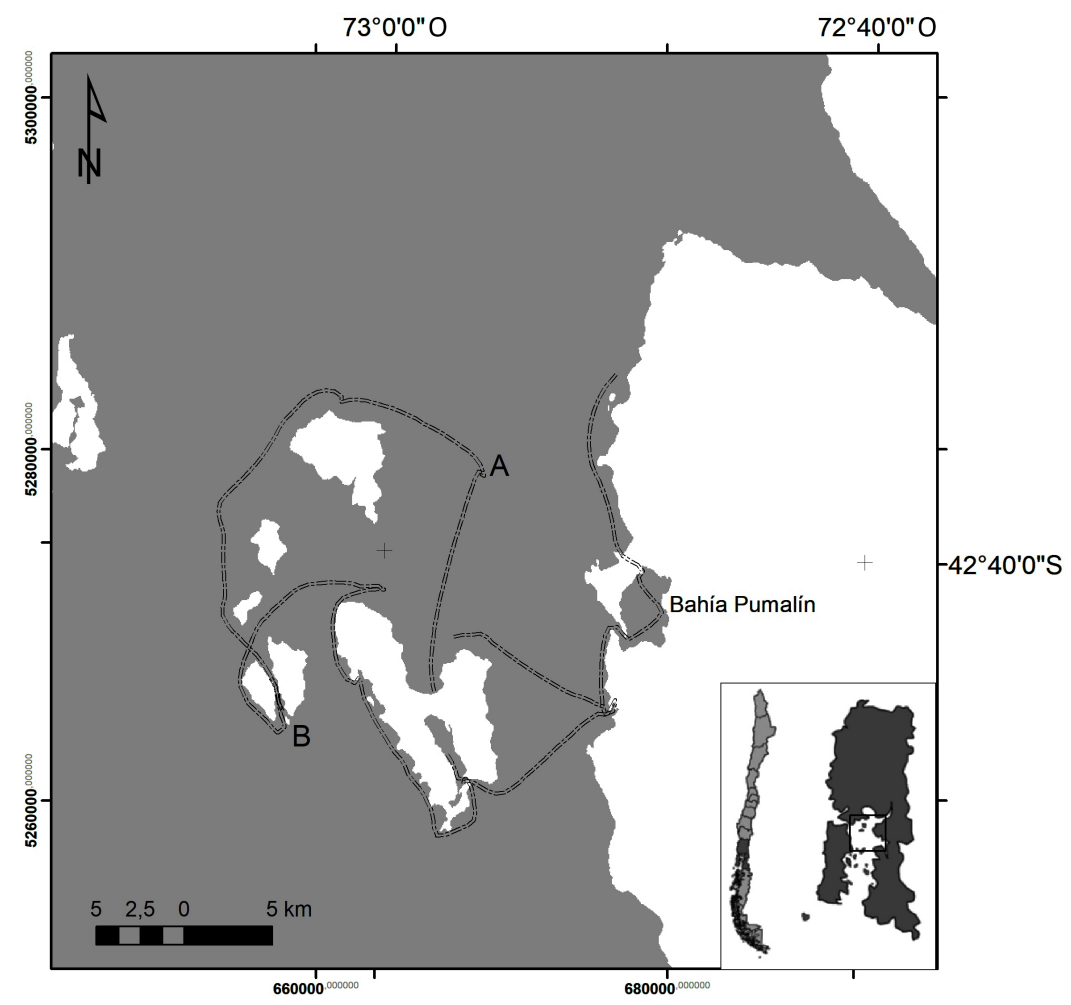

Fig. 1. Mapa con la ubicación espacial de los transectos de navegación, islote Nihuel (A) e islote Barraco (B), en el mar de islas Desertores, comuna de Chaitén, Patagonia chilena.

conteos directos de las aves y mamíferos marinos presentes. De forma similar se recorrió (1,5 $\mathrm{km})$ el entorno de islote Nihuel, apoyando las observaciones mediante lentes binoculares $(8 \times 42)$, GPS cartográfico (Garmin map) y cámaras fotográficas. Además, se utilizó dron (Dji) de resolución máxima $4 \mathrm{k}$, para fotografía aérea de colonias de nidificación ubicadas en zonas altas.

Las poblaciones de aves marinas nidificantes fueron evaluadas por conteo directo de los nidos aparentemente ocupados, los que se definieron como aquellos con capacidad para mantener los huevos y ocupados como mínimo por una especie de ave (Bibby et al. 2000). Para el caso de nidos del pingüino de Magallanes Spheniscus magellanicus (Foster, 1781), se consideró como nido activo también a las cuevas que presentaron signos de presencia como acumulación de plumones y fecas en su entrada.

Para cada especie registrada se identificó su condición de residente o migratoria según lo

\footnotetext{
1 http://especies.mma.gob.cl
}

propuesto por Iriarte (2010) y Couve et al. (2016), como así también su estado de conservación en base al criterio nacional $\left(\mathrm{MMA}^{1}\right.$ actualizado a diciembre de 2018) e internacional (IUCN ${ }^{2}$ ).

\section{RESULTADOS}

La composición taxonómica del total de aves observadas se estructuró en 10 órdenes y 19 familias, representadas por 39 especies (Tabla 2). De este grupo, el cisne de cuello negro Cygnus melancoryphus (Molina, 1782) es considerado En Peligro en la región de Los Lagos, mientras que la caranca Chloephaga hybrida (Molina, 1782) está clasificada como Vulnerable en todo el país. Otras cuatro especies [pingüino de Magallanes, albatros de ceja negra Thalassarche melanophris (Temminck, 1828), petrel gigante Macronectes giganteus (Gmelin, 1785), lile Phalacrocorax gaimardi (Lesson \& Garnot, 1828) y quetru no volador Tachyeres pteneres (Forster, 1844)]

2 https://www.iucnredlist.org 
Tabla 1. Detalle de los tramos que integraron los diferentes transectos de navegación realizados en el mar de islas Desertores, comuna de Chaitén, Patagonia chilena.

\begin{tabular}{ccccc}
\hline Tramo & Fecha & Horario & Coordenadas inicio & Coordenadas término \\
\hline A & $24-01-2019$ & $16: 28-17: 36$ & $42,57^{\circ} \mathrm{S} ; 72,84^{\circ} \mathrm{O}$ & $42,74^{\circ} \mathrm{S} ; 72,85^{\circ} \mathrm{O}$ \\
B & $24-01-2019$ & $18: 30-19: 02$ & $42,74^{\circ} \mathrm{S} ; 72,85^{\circ} \mathrm{O}$ & $42,78^{\circ} \mathrm{S} ; 72,93^{\circ} \mathrm{O}$ \\
C & $24-01-2019$ & $19: 12-19: 57$ & $42,78^{\circ} \mathrm{S} ; 72,94^{\circ} \mathrm{O}$ & $42,74^{\circ} \mathrm{S} ; 72,96^{\circ} \mathrm{O}$ \\
D & $25-01-2019$ & $11: 25-15: 36$ & $42,80^{\circ} \mathrm{S} ; 72,93^{\circ} \mathrm{O}$ & $42,58^{\circ} \mathrm{S} ; 73,03^{\circ} \mathrm{O}$ \\
E & $25-01-2019$ & $16: 00-17: 49$ & $42,68^{\circ} \mathrm{S} ; 73,01^{\circ} \mathrm{O}$ & $42,73^{\circ} \mathrm{S} ; 73,07^{\circ} \mathrm{O}$ \\
F & $26-01-2019$ & $09: 30-13: 52$ & $42,73^{\circ} \mathrm{S} ; 73,06^{\circ} \mathrm{O}$ & $42,62^{\circ} \mathrm{S} ; 72,93^{\circ} \mathrm{O}$ \\
G & $26-01-2019$ & $19: 39-20: 25$ & $42,62^{\circ} \mathrm{S} ; 72,93^{\circ} \mathrm{O}$ & $42,70^{\circ} \mathrm{S} ; 72,94^{\circ} \mathrm{O}$ \\
H & $27-01-2019$ & $10: 55-11: 20$ & $42,73^{\circ} \mathrm{S} ; 72,96^{\circ} \mathrm{O}$ & $42,73^{\circ} \mathrm{S} ; 72,88^{\circ} \mathrm{O}$ \\
\hline
\end{tabular}

están clasificadas como Cercanas a la Amenaza de extinción, mientras que la golondrina de mar Pincoya Oceanites pincoyae (Harrison, 2013) es considerada como con Datos Deficientes (Tabla 2). Mediante la navegación se determinó que las especies gaviota cahuil Chroicocephalus maculipennis (Lichtenstein, 1823), cormorán imperial Leucocarbo atriceps (King, 1828), gaviota dominicana Larus dominicanus (Lichtenstein, 1823), cisne de cuello negro y blanquillo Podiceps occipitalis (Garnot, 1826), fueron las aves más abundantes de observar (Tabla 3). Mientras que la gaviota de Franklin Leucophaeus pipixcan (Wagler, 1831) fue el ave migratoria con mayor abundancia en la zona (Tabla 3).

Tabla 2. Listado de las especies de aves y mamíferos marinos observados tanto en navegación como en tierra, en islas Desertores, comuna de Chaitén, Patagonia chilena. Indicando la condición (Map: migrante austral parcial, R: residente, Ma: migrante austral, Mb: migrante boreal) y estado de conservación (EC: criterio nacional/criterio internacional) de las especies observadas (EP: en peligro, VU: vulnerable, CA: cercana a la amenaza, MP: menor preocupación, DD: datos deficientes, Ni: no indica).

\begin{tabular}{|c|c|c|c|}
\hline Clasificación Taxonómica & Nombre común & Condición & E. C. \\
\hline \multicolumn{4}{|l|}{$\begin{array}{l}\text { Aves marino costeras } \\
\text { ORDEN SPHENISCIFORMES }\end{array}$} \\
\hline \multicolumn{4}{|l|}{ FAMILIA SPHENISCIDAE } \\
\hline Spheniscus magellanicus & Pingüino de Magallanes & Map & $\mathrm{Ni} / \mathrm{CA}$ \\
\hline \multicolumn{4}{|l|}{ ORDEN PODICIPEDIFORMES } \\
\hline \multicolumn{4}{|l|}{ FAMILIA PODICIPEDIDAE } \\
\hline Podiceps occipitalis & Blanquillo & $\mathrm{R}$ & $\mathrm{Ni} / \mathrm{MP}$ \\
\hline \multicolumn{4}{|l|}{ ORDEN PROCELLARIIFORMES } \\
\hline \multicolumn{4}{|l|}{ FAMILIA DIOMEDEIDAE } \\
\hline Thalassarche melanophris & Albatros de ceja negra & $\mathrm{R}$ & $\mathrm{MP} / \mathrm{CA}$ \\
\hline \multicolumn{4}{|l|}{ FAMILIA PROCELLARIIDAE } \\
\hline Macronectes giganteus & Petrel gigante & Ma & $\mathrm{Ni} / \mathrm{CA}$ \\
\hline \multicolumn{4}{|l|}{ FAMILIA HYDROBATIDAE } \\
\hline Oceanites oceanicus & Golondrina de mar & Ma & $\mathrm{Ni} / \mathrm{MP}$ \\
\hline Oceanites pincoyae & Golondrina de mar Pincoya & $\mathrm{R}$ & $\mathrm{Ni} / \mathrm{DD}$ \\
\hline
\end{tabular}




\begin{tabular}{|c|c|c|c|}
\hline Clasificación Taxonómica & Nombre común & Condición & E. C. \\
\hline \multicolumn{4}{|l|}{ ORDEN PELECANIFORMES } \\
\hline \multicolumn{4}{|l|}{ FAMILIA PHALACROCORACIDAE } \\
\hline Phalacrocorax brasilianus & Yeco & $\mathrm{R}$ & $\mathrm{Ni} / \mathrm{MP}$ \\
\hline Phalacrocorax gaimardi & Lile & $\mathrm{R}$ & $\mathrm{CA} / \mathrm{CA}$ \\
\hline Phalacrocorax magellanicus & Cormorán de las rocas & $\mathrm{R}$ & $\mathrm{Ni} / \mathrm{MP}$ \\
\hline Leucocarbo atriceps & Cormorán imperial & $\mathrm{R}$ & $\mathrm{Ni} / \mathrm{MP}$ \\
\hline \multicolumn{4}{|l|}{ ORDEN CICONIIFORMES } \\
\hline \multicolumn{4}{|l|}{ FAMILIA ARDEIDAE } \\
\hline Nycticorax nycticorax & Huairavo & $\mathrm{R}$ & $\mathrm{Ni} / \mathrm{MP}$ \\
\hline \multicolumn{4}{|l|}{ FAMILIA THRESKIORNITHIDAE } \\
\hline Theristicus melanopis & Bandurria & $\mathrm{R}$ & $\mathrm{Ni} / \mathrm{MP}$ \\
\hline \multicolumn{4}{|l|}{ FAMILIA CATHARTIDAE } \\
\hline Coragyps atratus & Jote & $\mathrm{R}$ & $\mathrm{Ni} / \mathrm{MP}$ \\
\hline Cathartes aura & Gallinazo & $\mathrm{R}$ & $\mathrm{Ni} / \mathrm{MP}$ \\
\hline \multicolumn{4}{|l|}{ ORDEN ANSERIFORMES } \\
\hline \multicolumn{4}{|l|}{ FAMILIA ANATIDAE } \\
\hline Cygnus melancoryphus & Cisne de cuello negro & Map & $\mathrm{EP} / \mathrm{MP}$ \\
\hline Chloephaga hybrida & Caranca & $\mathrm{R}$ & VU/MP \\
\hline Chloephaga poliocephala & Canquén & $\mathrm{R}$ & $\mathrm{Ni} / \mathrm{MP}$ \\
\hline Tachyeres pteneres & Quetru no volador & $\mathrm{R}$ & $\mathrm{CA} / \mathrm{MP}$ \\
\hline Anas georgica & Pato jergón & $\mathrm{R}$ & $\mathrm{Ni} / \mathrm{MP}$ \\
\hline \multicolumn{4}{|l|}{ ORDEN FALCONIFORMES } \\
\hline \multicolumn{4}{|l|}{ FAMILIA FALCONIDAE } \\
\hline Caracara plancus & Traro & $\mathrm{R}$ & $\mathrm{Ni} / \mathrm{MP}$ \\
\hline \multicolumn{4}{|l|}{ ORDEN CHARADRIIFORMES } \\
\hline \multicolumn{4}{|l|}{ FAMILIA HAEMATOPODIDAE } \\
\hline Haematopus palliatus & Pilpilén & $\mathrm{R}$ & $\mathrm{Ni} / \mathrm{MP}$ \\
\hline Haematopus ater & Pilpilén negro & $\mathrm{R}$ & $\mathrm{Ni} / \mathrm{MP}$ \\
\hline \multicolumn{4}{|l|}{ FAMILIA CHARADRIIDAE } \\
\hline Vanellus chilensis & Queltehue & $\mathrm{R}$ & $\mathrm{Ni} / \mathrm{MP}$ \\
\hline Charadrius modestus & Chorlo chileno & $\mathrm{Ma}$ & $\mathrm{Ni} / \mathrm{MP}$ \\
\hline \multicolumn{4}{|l|}{ FAMILIA SCOLOPACIDAE } \\
\hline Tringa melanoleuca & Pitotoy grande & $\mathrm{Mb}$ & $\mathrm{Ni} / \mathrm{MP}$ \\
\hline Numenius phaeopus & Zarapito & $\mathrm{Mb}$ & $\mathrm{Ni} / \mathrm{MP}$ \\
\hline
\end{tabular}




\begin{tabular}{|c|c|c|c|}
\hline Clasificación Taxonómica & Nombre común & Condición & E. C. \\
\hline Arenaria interpres & Playero vuelvepiedras & $\mathrm{Mb}$ & $\mathrm{Ni} / \mathrm{MP}$ \\
\hline Calidris bairdii & Playero de Baird & $\mathrm{Mb}$ & $\mathrm{Ni} / \mathrm{MP}$ \\
\hline Gallinago paraguaiae & Becacina & Map & $\mathrm{MP} / \mathrm{MP}$ \\
\hline \multicolumn{4}{|l|}{ FAMILIA LARIDAE } \\
\hline Larus scoresbii & Gaviota austral & $\mathrm{R}$ & $\mathrm{Ni} / \mathrm{MP}$ \\
\hline Chroicocephalus maculipennis & Gaviota cahuil & $\mathrm{Ma}$ & $\mathrm{Ni} / \mathrm{MP}$ \\
\hline Leucophaeus pipixcan & Gaviota de Franklin & $\mathrm{Mb}$ & $\mathrm{Ni} / \mathrm{MP}$ \\
\hline Larus dominicanus & Gaviota dominicana & $\mathrm{R}$ & $\mathrm{Ni} / \mathrm{MP}$ \\
\hline Sterna hirundinacea & Gaviotín sudamericano & $\mathrm{Ma}$ & $\mathrm{Ni} / \mathrm{MP}$ \\
\hline \multicolumn{4}{|l|}{ ORDEN APODIFORMES } \\
\hline \multicolumn{4}{|l|}{ FAMILIA TROCHILIDAE } \\
\hline Sephanoides sephaniodes & Picaflor común & $\mathrm{R}$ & $\mathrm{Ni} / \mathrm{MP}$ \\
\hline \multicolumn{4}{|l|}{ ORDEN PASSERIFORMES } \\
\hline \multicolumn{4}{|l|}{ FAMILIA FURNARIIDAE } \\
\hline Cinclodes patagonicus & Churrete común & Map & $\mathrm{Ni} / \mathrm{MP}$ \\
\hline Aphrastura spinicauda & Rayadito & Map & $\mathrm{Ni} / \mathrm{MP}$ \\
\hline \multicolumn{4}{|l|}{ FAMILIA HIRUNDINIDAE } \\
\hline Pygochelidon cyanoleuca & Golondrina de rabadilla negra & $\mathrm{R}$ & $\mathrm{Ni} / \mathrm{MP}$ \\
\hline \multicolumn{4}{|l|}{ FAMILIA TURDIDAE } \\
\hline Turdus falcklandii & Zorzal & $\mathrm{R}$ & $\mathrm{Ni} / \mathrm{MP}$ \\
\hline \multicolumn{4}{|l|}{$\underline{\text { Mamíferos marinos }}$} \\
\hline \multicolumn{4}{|l|}{ ORDEN CARNIVORA } \\
\hline \multicolumn{4}{|l|}{ FAMILIA MUSTELIDAE } \\
\hline Lontra felina & Chungungo & $\mathrm{R}$ & $\mathrm{VU} / \mathrm{EP}$ \\
\hline \multicolumn{4}{|l|}{ FAMILIA OTARIIDAE } \\
\hline Arctocephalus australis & Lobo fino austral & $\mathrm{R}$ & $\mathrm{CA} / \mathrm{MP}$ \\
\hline Otaria flavescens & Lobo marino común & $\mathrm{R}$ & $\mathrm{MP} / \mathrm{MP}$ \\
\hline \multicolumn{4}{|l|}{ ORDEN CETACEA } \\
\hline \multicolumn{4}{|l|}{ FAMILIA DELPHINIDAE } \\
\hline Cephalorhynchus eutropia & Delfín chileno & $\mathrm{R}$ & $\mathrm{VU} / \mathrm{CA}$ \\
\hline Lagenorhynchus australis & Delfín austral & $\mathrm{R}$ & $\mathrm{MP} / \mathrm{DD}$ \\
\hline
\end{tabular}


Tabla 3. Abundancia de las especies de aves y mamíferos marinos observados en islas Desertores, comuna de Chaitén, Patagonia chilena. Para detalle de los tramos ver Tabla 1.

\begin{tabular}{|c|c|c|c|c|c|c|c|c|c|}
\hline Especie & A & B & $\mathrm{C}$ & $\mathrm{D}$ & $E$ & $\mathrm{~F}$ & $G$ & $\mathrm{H}$ & Total \\
\hline Albatros de ceja negra & & & & & & 1 & & & 1 \\
\hline Bandurria & 2 & & 3 & & & & & & 5 \\
\hline Blanquillo & 28 & 123 & 20 & & 88 & 18 & & & 277 \\
\hline Canquén & & & 6 & & & & & & 6 \\
\hline Caranca & & & 16 & 12 & 11 & 2 & & & 41 \\
\hline Churrete común & & & 2 & & & & & & 2 \\
\hline Cisne de cuello negro & 650 & & 4 & & & & & & 654 \\
\hline Cormorán de las rocas & & & & 4 & 5 & 2 & & 2 & 13 \\
\hline Cormorán imperial & 4 & 10 & 13 & 90 & 129 & 169 & 9 & 11 & 435 \\
\hline Gallinazo & & & & 1 & & & & 1 & 2 \\
\hline Gaviota cahuil & 300 & 24 & 2 & & 32 & 154 & & 2 & 514 \\
\hline Gaviota de Franklin & 100 & 1 & & & 8 & & & 1 & 110 \\
\hline Gaviota dominicana & 250 & 1 & 28 & 36 & 74 & 11 & & & 400 \\
\hline Gaviotín sudamericano & 1 & 8 & & 1 & 20 & 1 & & & 31 \\
\hline Golondrina de mar & & & & & & 1 & & & 1 \\
\hline Golondrina de mar Pincoya & & & & & & 1 & & & 1 \\
\hline Huairavo & & 1 & & & & & & & 1 \\
\hline Lile & 1 & & & & & & & & 1 \\
\hline Pato jergón & 16 & & 60 & & & & & & 76 \\
\hline Petrel gigante & & & & & & 2 & & & 2 \\
\hline Pilpilén & 45 & & 8 & & 4 & 2 & & & 59 \\
\hline Pilpilén negro & & & 1 & & 3 & & & & 4 \\
\hline Pingüino de Magallanes & & & & 1 & 2 & 4 & 2 & & 9 \\
\hline Pitotoy grande & 1 & & & & & & & & 1 \\
\hline Queltehue & & & 4 & & & & & & 4 \\
\hline Quetru no volador & & & 22 & 16 & 44 & & & 4 & 86 \\
\hline Yeco & 4 & 7 & 5 & 2 & 1 & 1 & & & 20 \\
\hline Zarapito & 15 & & & & 25 & 2 & & & 42 \\
\hline Chungungo & & & & 1 & & & & & 1 \\
\hline Delfín austral & & & & & 10 & 2 & & & 12 \\
\hline Delfín chileno & & 1 & & & & & & & 1 \\
\hline Lobo marino común & & 1 & & 121 & & & & 1 & 123 \\
\hline Total & 1417 & 177 & 194 & 285 & 456 & 373 & 11 & 22 & 2935 \\
\hline
\end{tabular}


La composición taxonómica de mamíferos marinos se estructuró en dos órdenes y tres familias, representadas por cinco especies (Tabla 2). De este grupo, una especie [chungungo Lontra felina (Molina, 1782)] está clasificada como En Peligro de extinción y otras dos [lobo fino austral Arctocephalus australis (Zimmermann, 1783) y delfín chileno Cephalorhynchus eutropia (Gray, 1846)] como Cercana a la Amenaza. Mientras que el delfín austral Lagenorhynchus australis (Peale, 1848) es considerado como con Datos Deficientes (Tabla 2).

Durante la navegación se determinó que las especies lobo marino común Otaria flavescens (Shaw, 1800) y delfín austral, fueron los mamíferos marinos más abundantes de observar (Tabla 3).

A nivel espacial, las mayores concentraciones de aves y mamíferos marinos se encontraron en los islotes Nihuel y Barraco, como así también en el litoral de isla Talcán (Tabla 3).

El islote Nihuel, también conocido localmente como Piedra de Calto, destaca por su geomorfología y la variedad de hábitat que favorece el refugio y reproducción de aves $\mathrm{y}$ mamíferos marinos (Tabla 3 y 4). El centro de este islote consiste en un gran peñón de roca cuya zona alta posee una amplia superficie con poca pendiente, en cuya área nidifica un estimado de al menos 2.100 parejas de cormorán imperial (Tabla 4). Mientras que en su ladera norte nidifica lile (Tabla 4). En todo el contorno del peñón existe una densa vegetación boscosa y arbustiva dominada por chilco Fuchsia magellanica (Lam.), existiendo también otras especies vegetales como quila Chusquea quila (Kunth), chupallilla (Bromelia sp.), calafate Berberis microphylla (Forst), ortiga Urtica dioica L., calle-calle Libertia chilensis (Molina), que junto a otras plantas constituyen un atractivo hábitat para aves terrestres como rayaditos Aphrastura spinicauda (Gmelin, 1789), zorzales Turdus falcklandii (Quoy \& Gaimard, 1824) y picaflores Sephanoides sephaniodes (Lesson, 1827). Dentro de este cordón vegetal, los pingüinos de Magallanes construyen sus nidos en cuevas bajo tierra, existiendo una red de senderos utilizados por estas aves para desplazarse hacia el mar (Tabla 4). Mientras que en la zona supramareal del islote, se observó la nidificación de gaviota dominicana, gaviota austral Larus scoresbii (Traill, 1823) y pilpilén negro (Vieillot y Oudart 1825) (Tabla 4). La zona supramareal es dominada por rocas de diversos tamaños, que son utilizadas por lobos marinos para descanso y reproducción, destacando la alta abundancia del lobo fino austral, estimando la presencia de al menos 890 individuos.

Tabla 4. Abundancia reproductiva (número de nidos y polluelos) de aves marinas y costeras en islas Desertores, comuna de Chaitén, Patagonia chilena.

\begin{tabular}{|c|c|c|c|}
\hline Especie & sur isla Talcán & Islote Barraco & Islote Nihuel \\
\hline Cormorán de las rocas & 14 nidos activos & & \\
\hline Cormorán imperial & 1 nido activo & & 2100 nidos activos \\
\hline Gaviota austral & & & 16 polluelos \\
\hline Gaviota dominicana & & 54 polluelos & 255 polluelos \\
\hline \multirow{4}{*}{ Gaviotín sudamericano } & & 9 polluelos & \\
\hline & & 18 nidos con 1 huevo & \\
\hline & & 4 nidos con 1 huevo & \\
\hline & & 1 nido con 3 huevos & \\
\hline Lile & & & 17 nidos activos \\
\hline \multirow{2}{*}{ Pilpilén } & & 1 nido con 3 huevos & \\
\hline & & 1 nido con 1 polluelo y 1 huevo & \\
\hline Pilpilén negro & & & 1 nido con 2 huevos \\
\hline Pingüino de Magallanes & & & 28 nidos activos \\
\hline
\end{tabular}


Por otra parte, el islote Barraco destaca por ser una roca de aproximadamente una hectárea, que contiene una alta diversidad de aves costeras y marinas, registrando la nidificación de gaviota dominicana, gaviotín sudamericano Sterna hirundinacea (Lesson, 1831) y pilpilén Haematopus palliatus (Temminck, 1820) (Tabla 4). También se observó la presencia de aves migratorias como playero de Baird Calidris bairdii (Coues, 1861), playero vuelvepiedras Arenaria interpres (Linnaeus, 1758) y chorlo chileno Charadrius modestus (Lichtenstein, 1823).

Finalmente, isla Talcán destaca por poseer el mayor tamaño en la zona y en cuyo litoral se observó la presencia del chungungo y delfín chileno (Tabla 3). Para el grupo de aves acuáticas las especies blanquillo, caranca y pato jergón Anas georgica (Gmelin, 1789) destacaron en abundancia, observando un grupo de canquén Chloephaga poliocephala (Sclater, 1857) donde un individuo portaba un tubo plástico atrapado en su cuello.

\section{DISCUSIÓN}

La navegación en el mar de islas Desertores permitió determinar que las especies gaviota cahuil, cormorán imperial, gaviota dominicana, cisne de cuello negro y blanquillo, fueron las aves costeras más abundantes de observar durante la temporada estival. Estas especies forman parte del grupo de aves costeras dominantes del mar interior de Chiloé y ecorregión Chiloense en general (Imberti, 2005; Hucke-Gaete et al. 2010). Las gaviotas cahuil y dominicana se distribuyen ampliamente en América del Sur, mientras que el blanquillo y el cormorán imperial son especies endémicas de la Patagonia (BirdLife International, 2018).

Con respecto a la presencia de aves migratorias, la gaviota de Franklin presentó la mayor abundancia durante la navegación en islas Desertores, mientras que en el islote Barraco se observó la presencia del playero de Baird, playero vuelvepiedras y chorlo chileno. La gaviota de Franklin y el playero de Baird nidifican entre abril y agosto en América del Norte, migrando hacia el hemisferio sur durante su temporada no reproductiva (primavera y verano austral), alcanzando la costa pacífica de Sudamérica desde Ecuador a Chile (del Hoyo et al. 1996). El playero vuelvepiedras nidifica en el margen boreal del hemisferio norte (Groenlandia, Noruega, Rusia, Suecia), migrando hacia el sur durante la primavera y verano austral (del Hoyo et al. 1996). Mientras que el chorlo chileno nidifica durante primavera y verano austral en el extremo sur de Sudamérica, migrando hacia el norte durante el otoño e invierno austral (Kusch \& Marín, 2004). Cabe destacar que los seis individuos de chorlo chileno observados en islote Barraco presentaron plumaje reproductivo (i.e., pecho rojo castaño y abdomen blanco, separados por una gran banda negra), pero no se identificaron signos de su nidificación en el área.

La navegación en el mar de islas Desertores permitió determinar que las especies lobo marino común y delfín austral, fueron los mamíferos marinos más abundantes de observar durante la temporada estival. Estas especies forman parte del grupo de mamíferos marinos residentes del mar interior de Chiloé y ecorregión Chiloense en general (Hucke-Gaete et al. 2010; Viddi et al. 2010).

A nivel espacial, las mayores concentraciones de aves y mamíferos marinos se encontraron en los islotes Nihuel y Barraco, como así también en el litoral de isla Talcán. En la zona alta del islote Nihuel nidifican al menos 2.100 parejas de cormorán imperial, lo que convierte a este lugar en uno de los sitios reproductivos más importantes para dicha especie en Chile (Cursach et al. 2010; Kusch \& Marín, 2013). En la zona supramareal del islote Nihuel existe una colonia reproductiva de lobo fino austral, compuesta por al menos 890 individuos, lo que convierte a este lugar en el segundo sitio de importancia (luego de isla Guafo) para la reproducción del lobo fino austral en la región de Los Lagos (Oliva et al. 2007; Seguel \& Pavés, 2018). Por otra parte, el islote Barraco es utilizado por gaviotines sudamericanos como sitio de nidificación, junto a un interesante grupo de aves playeras migratorias. A la vez, en isla Talcán existen importantes bosques de huiro Macrocystis pyrifera (Agardh, 1820) en donde se registró la presencia de un chungungo. Este animal es considerado como casi exterminado en el mar interior de Chiloé, debido principalmente a su histórica caza ilegal, la destrucción de su hábitat y la contaminación (Sielfeld \& Castilla, 1999; Valqui, 2012). 
En la región de Los Lagos existen determinadas islas $e$ islotes costeros que son utilizados por aves y mamíferos marinos para su reproducción. Gran parte de éstos se ubican en la costa expuesta al océano Pacífico, y cada vez adquieren mayor interés para el desarrollo del ecoturismo (Skewgar et al. 2009; Cursach et al. 2011; Seguel \& Pavés, 2018). Mientras que en la zona del mar interior, recién durante la última década se han descrito nuevos sitios de reproducción (Häussermann et al. 2012). Así, el presente trabajo suma al conocimiento científico dos nuevos sitios de reproducción de aves y mamíferos marinos para el mar interior de la ecorregión Chiloense: islote Nihuel e islote Barraco.

Los resultados del presente estudio han permitido determinar el ensamble estival de aves y mamíferos marinos de islas Desertores, constituyéndose en los primeros publicados para la zona. Sin embargo, en términos de realizar un aporte para la planificación de la conservación de la biodiversidad marina del área, se debe realizar un seguimiento al menos estacional en un ciclo anual.

A partir de nuestros resultados se identifican las siguientes líneas de trabajo con respecto al estudio y la conservación de aves y mamíferos marinos de la comuna de Chaitén. La primera de ellas se enfoca en el estudio de aves migratorias tanto en islas Desertores como en humedales costeros continentales, como Chana y bahía Pumalín. En esta línea, se destaca el registro de chorlos chilenos con plumaje reproductivo en el islote Barraco, lo que es llamativo de observar para una especie que nidifica en el extremo austral de la Patagonia $\left(53-55^{\circ} \mathrm{S}\right)$ durante la temporada estival (Kusch \& Marín, 2004). Una segunda línea podría enfocarse en la contaminación por plásticos de diversos orígenes, y elementos de la industria acuícola, que están impactando a la fauna acuática local. Al respecto, se observó un canquén enmallado en plástico en isla Talcán. Finalmente, se propone evaluar la importancia de los bosques de algas marinas para la biodiversidad, y en particular el rol que juegan para una especie casi extinta como el chungungo.

Es importante señalar que la información obtenida puede ser utilizada en procesos de planificación para la conservación de áreas marinas protegidas locales, y que el valor biocultural del islote Nihuel cumple con todos los atributos para ser declarado como un área silvestre protegida por el Estado.

\section{AGRADECIMIENTOS}

Al apoyo del Grupo Patagonia Mar y Tierra, y el financiamiento del Programa Austral Patagonia de la Universidad Austral de Chile. A la ayuda brindada por Juan Cárdenas de isla Autení, Eric Mayorga de Chana, Corporación Ambiental del Uso del Borde Costero Yene Purrun We. A dos revisores anónimos de revista AIP.

\section{LITERATURA CITADA}

Aguayo, A., Acevedo, J., \& Vargas, R. (2006). Diversidad de mamíferos marinos en las aguas del archipiélago de Los Chonos (4339'S - 4550'S), XI región de Chile. Revista Ciencia y Tecnología del Mar, 29, 129-145.

Bibby, C., Burgess, N., Hill, D., \& Mustoe, S. (2000). Bird census techniques. London, England: Academic Press.

BirdLife International. (2018). The IUCN Red List of Threatened Species. Version 2018. https://www.iucnredlist.org/. Accesed 18 May 2019.

Couve, E., Vidal, C., \& Ruiz, J. (2016). Aves de Chile. Sus islas Oceánicas y península Antártica. Punta Arenas, Chile: Editorial FS Expeditions.

Cursach, J. A., Simeone, A., Matus, R., Soto, O., Schlatter, R., Tobar, C., \& Ojeda, J. (2010). Distribución reproductiva del cormorán imperial (Phalacrocorax atriceps) en Chile. Boletín Chileno de Ornitología, 16, 9-16.

Cursach, J. A., Rau, J. R., Ojeda, J., Vilugrón, J., Tobar, C., Oyarzún, C., Soto, O., \& Suazo, C. (2011). Diversidad de aves y mamíferos marinos en bahía San Pedro, costa de Purranque, centro-sur de Chile. Gayana, 75, 174-182.

De la Barrera, F., Reyes-Paecke, S., \& Meza, L. (2011). Análisis del paisaje para la evaluación ecológica rápida de alternativas de relocalización de una ciudad devastada. 
Revista Chilena de Historia Natural, 84, 181-194.

del Hoyo, J., Elliott, A., \& Sargatal, J. (1996). Handbook of the Birds of the World (vol. 3). Hoatzin to Auks. Barcelona, Spain: Lynx Edicions.

Häussermann, V., Forsterra, G., \& Plotnek, E. (2012). Sightings of marine mammals and birds in the Comau Fjord, Northern Patagonia, between 2003 and mid 2012 (Mammalia; Aves). Spixiana, 35, 247-262.

Hucke-Gaete, R., Ruiz, R., \& Álvarez, R. (2010). Descripción de la ecorregión Chiloense. In R. Hucke-Gaete, P. Lo Moro, \& J. Ruiz (Eds.), Conservando el mar de Chiloé, Palena y Guaitecas (pp. 26-62). Puerto Montt: Comisión Nacional del Medio Ambiente.

Imberti, S. (2005). Distribución otoñal de aves marinas y terrestres en los canales chilenos. Anales del Instituto de la Patagonia, 33, 21-30.

Iriarte, J. L., González, H. E., Liu, K. K., Rivas, C., \& Valenzuela, C. (2007). Spatial and temporal variability of chlorophyll and primary productivity in surface waters of southern Chile $\left(41.5-43^{\circ} \mathrm{S}\right)$. Estuarine, Coastal and Shelf Science, 74, 471-480.

Iriarte, A. (2010). Guía de campo de los mamíferos de Chile. Santiago, Chile: Editorial Flora y Fauna Chile Ltda.

Kusch, A., \& Marín, M. (2004). Distribución del chorlo chileno, Charadrius modestus, (Lichtenstein) (Charadriidae) en Chile. Anales del Instituto de la Patagonia, 32, 69-78.

Kusch, A., \& Marín, M. (2013). Distribución de sitios reproductivos de cormoranes Phalacrocorax spp. (Pelecaniformes) en el Estrecho de Magallanes y costas hacia el sur $\left(52^{\circ}-56^{\circ} \mathrm{S}\right)$, Chile. Anales del Instituto de la Patagonia, 41, 131-139.

Luebert, F., \& Pliscoff, P. (2006). Sinopsis bioclimática y vegetacional de Chile. Santiago, Chile: Editorial Universitaria.

Oliva, D., Sielfeld, W., Buscaglia, M, Matamala, M., Moraga, R., Pavés, H., Pérez, M. J.,... \& Sepúlveda, M. (2007). Plan de acción para disminuir y mitigar los efectos de las interacciones del lobo marino común
(Otaria flavescens) con las actividades de pesca y acuicultura de la X y XI región. Informe Final FIP $N^{\circ}$ 2006-34. http:// www.fip.cl/FIP/Archivos/pdf/informes/ inffinal 2006-34.pdf. Accesed 28 October 2019.

Palma, S., \& Silva, N. (2004). Distribution of siphonophores, chaetognaths, euphausiids and oceanographic conditions in the fjords and channels of southern Chile. DeepSea Research Part II: Topical Studies in Oceanography, 51, 513-535.

Palma, S., Silva, N., Retamal, M. C., \& Castro, L. (2011). Seasonal and vertical distributional patterns of siphonophores and medusae in the Chiloé Interior Sea, Chile. Continental Shelf Research, 31, 260-271.

Ramírez, B., \& Pizarro, E. (2005). Distribución de clorofila a y feopigmentos en los canales australes chilenos comprendidos entre Puerto Montt y la laguna San Rafael, Chile. Ciencia y Tecnología del Mar, 28, 45-62.

Seguel, M., \& Pavés, H. (2018). Sighting patterns and habitat use of marine mammals at Guafo Island, Northern Chilean Patagonia during eleven austral summers. Revista de Biología Marina y Oceanografía, 53, 237-250.

Sielfeld, W., \& Castilla, J.C. (1999). Estado de conservación y conocimiento de las nutrias en Chile. Estudios Oceanológicos, 18, 6979.

Skewgar, E., Simeone, A., \& Boersma, P. (2009). Marine Reserve in Chile would benefit penguins and ecotourism. Ocean \& Coastal Management, 52, 487-491.

Sullivan, K., \& Bustamante, G. (1999). Setting geographic priorities for marine conservation in Latin America and the Caribbean. Arlington, USA: The Nature Conservancy.

Tasker, M., Hope Jones, P., Dixon, T., \& Blake, B. (1984). Counting seabirds at sea from ships: a review of methods employed and a suggestion for a standardized approach. The Auk, 101, 567-577.

Valqui, J. (2012). The marine otter Lontra felina (Molina, 1782): A review of its present status and implications for future conservation. Mammalian Biology, 77, 75-83. 
Viddi, F. A., Hucke-Gaete, R., Torres-Florez, J. P., \& Ribeiro, S. (2010). Spatial and seasonal variability in cetacean distribution in the fjords of northern Patagonia, Chile. ICES Journal of Marine Science, 67, 959-970. 